\title{
Dislocation Emission and Cleavage Process at Crack Tip
}

\author{
T.-C. Wang \\ LNM, Institute of Mechanics, Chinese Academy of Science, Beijing 100080, China \\ Keywords: Dislocation Emission, Cleavage, Mode I Crack, Cohesive Zone
}

\begin{abstract}
The competition process between dislocation emission along a pair of symmetric slip planes and quasi-cleavage at Mode I crack tip is investigated in this paper. The equilibrium positions and the total number of emitted dislocations are determined based on Peierls framework. The fundamental physical events at the crack tip are reviewed. The crack blunting effects on the stress fields near the top of the blunted crack are analysed.
\end{abstract}

\section{INTRODUCTION}

During the last two decades much experimental and theoretical work has been published concerning the brittle versus ductile behaviours of materials. The fundamental physical events at a crack tip include dislocation nucleation, dislocation emission and crack cleavage. The process of dislocation emission from a stressed crack tip has been observed by many authors $[1,2,3]$. Rice and Thomson[4] presented a dislocation emission model to characterize the plastic shear at a crack tip. Rice[5], Schoeck[6] and Rice et al.[7] have reanalyzed the Rice-Thomson criterion on the basis of the Peierls concept.

A new approach was developed by Wang[8] with a slightly modified Rice's model. Both the dislocation nucleation and emission from the crack tip were analysed. A unified model for dislocation nucleation, dislocation emission and dislocation free zone has been proposed by Wang[9]. A general theory of brittle fracture criterion accounting for the effects of the dislocation emission has been developed by Wang[10].

Recent experiments[11,12] have revealed a new mechanism of quasi-cleavage fracture by forming a nanocrack in the Dislocation Free Zone and by linking the nanocrack with the main crack. Zhu et al.[13] have analyzed the quasi-cleavage process driven by dislocation pileups.

The competition process between dislocation emission and quasi-cleavage at a Mode I crack tip is investigated in this paper. The dislocation can emit on a pair of symmetric slip planes of angle $\alpha$ with respect the crack plane. The equilibrium positions and the total number of emitted dislocations are determined by means of a Peierls-type stress versus displacement relation in the cohesive zone. The attention will focus on the fundamental physical events at the crack tip. The strong interaction between the cohesive zone and the emitted dislocation is revealed as the DFZ size is small. The crack blunting effects on the stress fields near the top of the blunted crack are analysed. A fracture criterion after a number of dislocation emissions is proposed. 


\section{Dislocation Nucleation and Emission along inclined planes}

We consider the process of dislocation nucleation and emission on a pair of symmetric slip planes of angle $\alpha$ with respect to the crack plane as shown in Fig.1. The crack configuration is remotely loaded by pure mode I stress intensity factor $K_{I}$. The integer $N_{d}$ denotes the total number of emitted dislocation in one arm of the symmetric slip planes. The equilibrium positions of the emitted dislocation are denoted by $r_{i}, i=1,2, \cdots N_{d}$.

Assume the emitted dislocations on each arm pile up behind an obstacle, which is located at $r_{o b}$. Hence we have a constraint equation $r_{1}=r_{o b}$.

The dislocation nucleation and emission are treated as a pure shear process. An incipient static distribution $\delta_{r}$ of sliding discontinuity across a pair of symmetric slip planes develops in the cohesive zones. The equilibrium equation for shear along upper slip planes in the cohesive zone is

$$
\tau\left[\Delta_{r}\right]=\tau_{r \theta}^{(0)}(r)+A \int_{0}^{R} g(r, \rho) b_{r}(\rho) d \rho+A b \sum_{j=1}^{n} g\left(r, r_{j}\right)
$$

where $\mathrm{R}$ is the length of the cohesive zone, $A=\mu /(\kappa+1) \pi, n=N_{d}$, and $\tau_{r \theta}^{(0)}(r)$ is the singular shear stress due to the applied stress intensity factor $K_{I}$

$$
\tau_{r \theta}^{(0)}(r)=\frac{K_{I} b}{2 \sqrt{2 \pi r}} \sin \alpha \cos \frac{\alpha}{2}
$$

where $b$ is the magnitude of the Burgers vector along the inclined slip planes.

The second term in the right hand of Eq.(1) is the shear stress produced by the distributed dislocations in the upper and lower cohesive zones. The third term is the shear stress contributed by the emitted discrete dislocations in the plastic zones. $\tau\left[\Delta_{r}\right]$ is the cohesive shear stress,

$$
\begin{aligned}
& \tau\left[\Delta_{r}\right]=\tau_{\max } \sin \left(2 \pi \frac{\Delta_{r}}{b}\right) \\
& \Delta_{r}=\delta_{r}+\frac{\tau}{\mu} h
\end{aligned}
$$

where $\mathrm{h}$ is the interplanar spacing across the slip plane.

For the $\mathrm{i}$-th emitted dislocation in the upper plastic zone, we have

$$
\tau_{r \theta}^{(0)}\left(r_{i}\right)+A \int_{0}^{R} g\left(r_{i}, \rho\right) b_{r}(\rho) d \rho+A b \sum_{j=1}^{n} g\left(r_{i}, r_{j}\right)+A b\left[g_{0}\left(r_{i}, r_{i}\right)+g_{1}\left(r_{i}, r_{i}\right)\right]=\tau_{f}, i=2,3, \cdots, N_{d}
$$

where

$$
g(r, \rho)=\frac{2}{r-\rho}+g_{0}(r, \rho)+g_{1}(r, \rho)
$$

The detailed formulae of the functions $g_{0}(r, \rho)$ and $g_{1}(r, \rho)$ can be obtained from the following derivation.

Stress and displacement components can be expressed by complex potentials $\Phi(z)$ and $\Omega(z)$,

$$
\begin{aligned}
& \sigma_{11}+\sigma_{22}=4 \operatorname{Re}\{\Phi(z)\} \\
& \sigma_{22}+i \tau_{12}=\overline{\Phi(z)}+\Omega(z)+(\bar{z}-z) \Phi^{\prime}(z) \\
& 2 \mu(u-i u)_{x}^{\prime}=\kappa \Phi(z)-\Omega(z)-(\bar{z}-z) \Phi^{\prime}(z)
\end{aligned}
$$

The interaction problem between a crack and dislocations has been studied by many authors (see Lin and Thomson[14] and Suo[15]). The solution can be obtained by the superpositions of 
two solutions. One corresponds to a dislocation solution in an infinite plate without the crack. The second solution is due to the interaction between the crack and the dislocation. Hence we have

$$
\begin{aligned}
& \Phi(z)=\Phi_{0}(z)+\Phi_{1}(z) \\
& \Omega(z)=\Omega_{0}(z)+\Omega_{1}(z)
\end{aligned}
$$

The potentials $\Phi_{0}(z)$, and $\Omega_{0}(z)$ for a pair of dislocations at $s$ and $\bar{s}$ are given by

$$
\begin{aligned}
& \Phi_{0}(z, s)=\frac{B}{z \overline{\bar{B}} s}+\frac{\bar{B}}{z-\bar{s}} \\
& \Omega_{0}(z, s)=\frac{B(\bar{s}-s)}{z-s}+\frac{B}{(z-s)^{2}}+\frac{\bar{B}(s-\bar{s})}{z-\bar{s}}+\frac{(z-\bar{s})^{2}}{(z-5)}
\end{aligned}
$$

where

$$
B=\frac{\mu}{\pi(\kappa+1) i}\left(b_{1}+i b_{2}\right)=\frac{\mu e^{i \alpha}}{\pi(\kappa+1) i}\left(b_{r}+i b_{\theta}\right)
$$

$b_{1}$ and $b_{2}$ are the Burgers vector components in $x_{1}$ and $x_{2}$ directions, respectively, $\kappa=3-4 \nu$ for plane strain.

The second potentials $\Phi_{1}(z)$ and $\Omega_{1}(z)$ can be expressed as

$$
\begin{gathered}
\Omega_{1}(z)=\bar{\Phi}_{1}(z)=-\frac{1}{2}\{(B+\bar{B})[F(z, s)+F(z, \bar{s})] \\
+(s-\bar{s})\left[B F_{1}(z, s)-\bar{B} F_{1}(z, \bar{s}]\right\}
\end{gathered}
$$

where

$$
F(z, s)=\frac{1}{z-s}\left[1-\sqrt{\frac{s}{z}}\right], s F_{1}(z, s)=z \frac{\partial F(z, s)}{\partial z}+F(z, s)
$$

In our situation $b_{\theta}=0, b_{r}=b$.

Using Eq.(10), Eq.(9) can be written as

$$
\Omega_{1}(z, s)=\bar{\Phi}_{1}(z, s)=-A b \sin \alpha\left\{z \frac{\partial}{\partial z}[F(z, s)+F(z, \bar{s})]+2[F(z, s)+F(z, \bar{s})]\right\}
$$

Regarding to the contribution of the continuous distribution of edge dislocations in the upper and lower cohesive zones, we have

$$
\begin{aligned}
& \Phi^{c}(z)=\Phi_{0}^{c}(z)+\Phi_{1}^{c}(z) \\
& \Omega^{c}(z)=\Omega_{0}^{c}(z)+\Omega_{1}^{c}(z)
\end{aligned}
$$

Potential $\Phi_{0}^{c}(z)$ takes the form

$$
\begin{aligned}
\Phi_{0}^{c}(z) & =\pi A i\left[G(\zeta)-G\left(\zeta_{*}\right)\right] \\
G\left(\zeta_{*}\right) & =\frac{1}{\pi} \int_{0}^{R} \frac{e^{i \alpha}}{z-s} b_{r}(\rho) d \rho \\
G(\zeta) & =\frac{1}{\pi} \int_{0}^{R} \frac{e^{-i \alpha}}{z-s} b_{r}(\rho) d \rho
\end{aligned}
$$

where $b_{\boldsymbol{r}}(\rho)$ is the dislocation density, $s=\rho e^{i \alpha}$,

$$
\zeta=\frac{2 z}{R} \epsilon^{i \alpha}-1, \quad \zeta_{*}=\frac{2 z}{R} e^{-i \alpha}-1
$$

The reason of introducing the variables $\zeta$ and $\zeta_{*}$ becomes clear later. Similarly, we have

$$
\Omega_{0}^{c}(z)=\Phi_{0}^{c}(z)+2 \pi A\left\{\sin \alpha\left(\frac{2 z}{R}\right)\left[e^{2 i \alpha} G^{\prime}(\zeta)+e^{-2 i \alpha} G^{\prime}\left(\zeta_{*}\right)\right]-i\left[e^{2 i \alpha} G(\zeta)-e^{-2 i \alpha} G\left(\zeta_{*}\right)\right]\right\}
$$


Furthermore, we obtain

$$
\bar{\Phi}_{1}^{c}(z)=\Omega_{1}^{c}(z)=-A \sin \alpha\left\{z\left[P^{\prime}(z)+Q^{\prime}(z)\right]+2[P(z)+Q(z)]\right\}
$$

where

$$
P(z)=\int_{0}^{R} F(z, s) b_{r}(\rho) d \rho, \quad Q(z)=\int_{0}^{R} F(z, \bar{s}) b_{r}(\rho) d \rho
$$

The singularity of the dislocation density $b_{r}(\rho)$ is less then $\frac{1}{\sqrt{\rho}}$ at the crack tip, while the dislocation density $b_{r}(\rho)$ vanishes at the end of the cohesive zone. With the variable translation $\rho=\frac{R}{2}(1+\cos \theta), 0 \leq \theta \leq \pi$, the dislocation density $b_{\mathbf{r}}(\rho)$ can be expressed as the following sine series

$$
b_{r}(\rho)=\sum_{m=1,2}^{\infty} \alpha_{m} \sin \left(m-\frac{1}{2}\right) \theta
$$

Then we have

$$
\sqrt{\frac{\rho}{R}} b_{r}(\rho)=\frac{1}{2} \sum_{m=1}^{\infty} \alpha_{m}[\sin m \theta+\sin (m-1) \theta]
$$

Substituting Eqs.(17) and (18) into Eqs.(13) and (15), it follows

$$
\begin{aligned}
& G\left(\zeta_{*}\right)=-\frac{1}{\pi} \int_{-1}^{1} \frac{b_{r}(\rho) d \xi}{\xi-\zeta_{*}}=\sum_{m=1}^{\infty} \alpha_{m} G_{m-\frac{1}{2}}\left(\zeta_{*}\right) \\
& P(z)=\sum_{m=1}^{\infty} \alpha_{m} P_{m}(z)+\frac{\pi}{2} \alpha_{1} e^{-\frac{i \alpha}{2}} \sqrt{\frac{R}{z}} \\
& Q(z)=\sum_{m=1}^{\infty} \alpha_{m} Q_{m}(z)+\frac{\pi}{2} \alpha_{1} e^{\frac{i \alpha}{2}} \sqrt{\frac{R}{z}}
\end{aligned}
$$

where

$$
\begin{aligned}
& P_{m}(z)=\pi e^{-i \alpha} G_{m-\frac{1}{2}}\left(\zeta_{*}\right)\left[1-\frac{h_{+}\left(\zeta_{*}\right)}{\sqrt{2} \sqrt{\zeta * 1}}\right] \\
& Q_{m}(z)=\pi e^{i \alpha} G_{m-\frac{1}{2}}(\zeta)\left[1-\frac{h_{+}(\zeta)}{\sqrt{2} \sqrt{\zeta+1}}\right] \\
& h_{+}(\zeta)=\left(\zeta-\sqrt{\zeta^{2}-1}\right)^{1 / 2}+\left(\zeta-\sqrt{\zeta^{2}-1}\right)^{-1 / 2}
\end{aligned}
$$

In derivation above equations, the following formula ${ }^{[8]}$ is used

$$
G_{m}(\zeta)=-\frac{1}{\pi} \int_{-1}^{1} \frac{\sqrt{1-\zeta^{2}} \cdot U_{m}(\xi) d \xi}{\xi-\zeta}=\left[\zeta-\sqrt{\zeta^{2}-1}\right]^{m}
$$

where $U_{m}(\xi)$ is the second Chebyshev polynomial

$$
U_{m}(\xi)=\frac{\sin m \theta}{\sin \theta}, \quad \xi=\cos \theta, \quad m=1,2, \cdots
$$

The sliding displacement takes the form

$$
\delta_{\boldsymbol{r}}=\int_{r}^{R} b_{\boldsymbol{r}}(\rho) d \rho=-\frac{R}{4} \sum_{m=1,2}^{\infty} \alpha_{m}\left[\frac{\sin \left(m+\frac{1}{2}\right) \theta}{\left(m+\frac{1}{2}\right)}-\frac{\sin \left(m-\frac{3}{2}\right) \theta}{\left(m-\frac{3}{2}\right)}\right]
$$

The shear stress $\tau_{r \theta}$ along the upper slip plane is

$$
\tau_{r \theta}=\operatorname{Im}\left\{e^{2 i \alpha}\left[\Omega(z)-\Phi(z)+(\bar{z}-z) \Phi^{\prime}(z)\right]\right\}
$$

In our situation, the emitted dislocations in the plastic zones are treated as two sets of discrete elastic edge dislocations. The cohesive zones immediately ahead of the crack tip along 
a pair of symmetric slip planes are considered as continuous distributions of infinitesimal edge dislocations.

Hence the total potentials $\Phi(z)$ and $\Omega(z)$ can be expressed as

$$
\begin{aligned}
\Phi(z)= & \Phi^{(0)}(z)+\pi A i \sum_{m=1}^{\infty} \alpha_{m}\left[G_{m-\frac{1}{2}}(\zeta)-G_{m-\frac{1}{2}}\left(\zeta_{*}\right)\right] \\
& -A \sin \alpha\left(z \frac{\partial}{\partial z}+2\right)\left\{\sum_{m=1}^{\infty} \alpha_{m}\left[P_{m}(z)+Q_{m}(z)\right]+\pi \alpha_{1} \cos \alpha \sqrt{\frac{R}{z}}\right\} \\
& -A b i \sum_{j=1}^{n}\left\{\frac{e^{i \alpha}}{z-z_{j}}-\frac{e^{-i \alpha}}{z-\bar{z}_{j}}\right\}-A b \sin \alpha\left(z \frac{\partial}{\partial z}+2\right) \sum_{j=1}^{n}\left[F\left(z, z_{j}\right)+F\left(z, \bar{z}_{j}\right)\right] \\
\Omega(z)= & \Phi(z)+2 \pi A \sum_{m=1}^{\infty} \alpha_{m}\left\{-i\left[e^{2 i \alpha} G_{m-\frac{1}{2}}(\zeta)-e^{-2 i \alpha} G_{m-\frac{1}{2}}\left(\zeta_{*}\right)\right]\right. \\
& \left.+\sin \alpha \frac{2 z}{R}\left[e^{2 i \alpha} G_{m-\frac{1}{2}}^{\prime}(\zeta)+e^{-2 i \alpha} G_{m-\frac{1}{2}}^{\prime}\left(\zeta_{*}\right)\right]\right\} \\
& +2 A b i \sum_{j=1}^{n}\left\{\cos \alpha\left[\frac{1}{z-z_{j}}-\frac{1}{z-\bar{z}_{j}}\right]+i r_{j} \sin \alpha\left[\frac{e^{i \alpha}}{\left(z-z_{j}\right)^{2}}+\frac{e^{-i \alpha}}{\left(z-\bar{z}_{j}\right)^{2}}\right]\right\}
\end{aligned}
$$

where $\Phi^{(0)}(z)$ is the potential of the $K$ field, $z_{j}=r_{j} e^{i \alpha}$ is the position of the $\mathrm{j}$-th emitted dislocation in the upper slip plane.

Using Eqs.(25) and (26), one can obtain the Eq.(1). After some manipulations, it follows

$$
\begin{aligned}
& g_{0}(r, \rho)=\operatorname{Re}\left\{2 e^{4 i \alpha}\left[\frac{1}{\left(\rho-r e^{2 i \alpha}\right)}+i \sin (2 \alpha) \frac{r}{\left(\rho-r e^{2 i \alpha}\right)^{2}}\right]\right\} \\
& g_{1}(r, \rho)=2 \sin ^{2} \alpha \operatorname{Re}\left\{e^{i \alpha} z \frac{\partial}{\partial z} \Phi_{R}(z, s)\right\}
\end{aligned}
$$

where

$$
\begin{aligned}
& \Phi_{R}(z, s)=\left(z \frac{\partial}{\partial z}+2\right)[F(z, s)+F(z, \bar{s})] \\
& z=r e^{i \alpha}, \quad s=\rho e^{i \alpha}
\end{aligned}
$$

The governing equations (1) and (4) can be written as

$$
\begin{gathered}
\tau_{r \theta}^{(0)}(r)+A b \sum_{m=1}^{\infty} \alpha_{m} T_{m}(r)+A b \sum_{j=1}^{n} g\left(r, r_{j}\right)=\tau_{\max } \sin \left(2 \pi \frac{\Delta_{r}}{b}\right), 0 \leq r \leq R \\
\tau_{r \theta}^{(0)}\left(r_{i}\right)+A b \sum_{m=1}^{\infty} \alpha_{m} T\left(r_{i}\right)+A b\left\{\sum_{j=1}^{n}{ }^{\prime} g\left(r_{i}, r_{j}\right)+g_{0}\left(r_{i}, r_{i}\right)+g_{1}\left(r_{i}, r_{i}\right)\right\}=\tau_{f}, i=2, \cdots, N_{d}
\end{gathered}
$$

where

$$
T_{m}(r)=\frac{1}{b} \int_{0}^{R} g(r, \rho) \sin \left(m-\frac{1}{2}\right) \theta d \rho
$$

The unknown coefficients $\left\{\alpha_{m}\right\}$ and $\left\{r_{i}\right\}$ are chosen as the basic unknown quantities. The infinite series in Eqs.(29) and (30) can be approximated with a sufficient degree of accuracy by the corresponding truncated series.

The cohesive zone $0 \leq r \leq R$ is discretized into $M$ elements, which vary in size along the region. The nodal points are given by the following expression

$$
r_{k}^{*}=\frac{R}{2}\left[1+\cos \left(\frac{(k-1) \pi}{M}\right)\right], k=1,2, \cdots, M
$$

The governing equations are then transformed into a set of nonlinear algebraic equations and solved by the Newton-Raphson method. 
The calculations were carried out with materials parameters $h / b=1.0, \nu=0.3, \tau_{f} / \mu=$ $0.002, \tau_{\max } / \mu=0.1596$, and $\alpha=45^{\circ}$.

Fig.2(a) shows variation of local stress intensity factor $K_{I}^{\text {tip }}$ versus $\delta_{r}^{\text {tip }}$ before dislocation emission. Fig.2(b) shows variation of local stress intensity factor $K_{I}^{\text {tip }}$ versus $\Delta_{r}^{t i p} / b-N_{d}$ after the $N_{d}$-th dislocation emission. It is presumed that the nucleated dislocation will emit out of the crack tip region when $K_{I}^{\text {tip }}$ reaches the maximum value.

The relation between the local stress intensity factor $K_{I}^{\text {tip }}$ and the applied stress intensity factor $K_{I}$ is shown in Fig.3. At the first stage, $K_{I}^{\text {tip }}$ is identical to $K_{I}$ before any dislocation emission. At point $A_{0}$, the local stress intensity factor $K_{I}^{\text {tip }}$ reaches the critical value $K_{I e}^{r(0)}$, then the first nucleated dislocation is emitted away from the crack tip. The $K_{I}^{\text {tip }}$ is decreased from point $A_{0}$ to $B_{1}$ along a vertical line $A_{0} B_{1}$. Since the emitted dislocation escapes rapidly from the crack tip with high speed, so that the applied stress intensity $K_{I}$ can be considered keeping constant during the dynamic process of dislocation emission.

As the applied stress intensity increases again, the $K_{I}^{\text {tip }}$ will increase along the straight line $B_{1} A_{1}$. At point $A_{1}$, the local stress intensity factor $K_{I}^{\text {tip }}$ achieves a critical value $K_{I e}^{(1)}$, which is only a little bit larger than $K_{I}^{(0)}$, the second dislocation will move ont of the crack tip region and stop at distance $r_{2}$. Then the local stress intensity factor $K_{I}^{\text {tip }}$ drops from point $A_{1}$ to point $B_{2}$ due to shielding by the second emitted dislocation.

The $K_{I e}^{(1)}$ is the maximum value of $K_{I}^{t i p}$ in a curve of $K_{I}^{t i p}$ versus $\left(\delta_{r}^{t i p} / b-1\right)$ after the first dislocation emission. As the sequence is repeated, at point $A_{N}$, the $K_{I}^{- \text {tip }}$ is exceeded the $K_{I}^{\text {intrim }}$, cleavage will occur before the next dislocation emission. Here the $K_{I}^{\text {intrin }}$ is the intrinsic fracture toughness of a dislocation free material.

The applied stress intensity factor $K_{I}$ versus the number $N_{d}$ is plotted in Fig.4. The dashed line between points $A_{i-1}$ to $B_{i}$ represents the dynamic process of the i-th dislocation emission. The solid vertical line $B_{i} A_{i}$ denotes the loading process after the i-th dislocation emission. Fig.5 shows the upper envelope of the actual curve of the applied stress intensity factor $K_{I}$ versus the total number $N_{d}$ of the emitted dislocation. A corresponding upper envelope curve for $K_{I}^{\text {tip }}$ is shown in Fig.6. We should emphasize that the upper envelope of the local stress intensity $K_{I}^{\text {tip }}$ increases very slowly with the increase of the total number $N_{d}$, meanwhile the upper envelope of the applied stress intensity factor $K_{I}$ increases rapidly.

\section{Crack Blunting Effects on Stress Distribution}

After numerous dislocation emission, the crack tip will blunt, which results in reduction of the stress fields ahead of the crack tip. For the sake of simplification, we assume that the blunted crack tip is a slot with circular top of radius $\rho=n b \sin \gamma / \cos \beta$. The angles $\beta$ and $\gamma$ are introduced in a simplified slip model as shown in Fig.7(b). The slot length $2 \mathrm{a}$ is extremely large compared with $b$ the length of the Burgers vector. In our situation, the length a is chosen to be about $100,000 \mathrm{~b}$.

According to Neuber's concept, the stress fields near the top of a deep slot are controlled by the slot length $2 \mathrm{a}$ and the curvature radius $\rho$ at the end of the top. The actual shape of the deep slot has only negligible effect on the stress fields near the top of the slot. Hence the deep slot can be treated as an elliptic notch with same length $2 a$ and same curvature radius at the end of the major axial.

Introduce mapping function $\omega(\xi)$

$$
\omega(\xi)=c\left(\xi+\frac{m}{\xi}\right)
$$


where

$$
c=\frac{1}{2}\left(a+b_{0}\right), m=\frac{\left(a-b_{0}\right)}{\left(a+b_{0}\right)}, b_{0}^{2}=a \rho
$$

Then the infinite region outside the elliptic notch translates into an unit circle and its exterior in the imagine plane. The infinite elastic plane with an elliptic notch subjects remote biaxial loading $\sigma_{x}^{\infty}=\sigma_{y}^{\infty}=\sigma_{0}$ and contains two pairs of emitted dislocation arrays as shown in Fig.7. The cohesive zones are neglected and the positions of emitted dislocations are assumed to be given by the method of last section after neglecting the cohesive zones. The stress field is composed of the applied remote stress field $\sigma_{i j}^{(0)}$, the stress field of the emitted dislocation $\sigma_{i j}^{(d)}$ and the image stress field due to the free surface of the blunted crack. The image stress field can be represented by the additional potentials $\phi(\xi)$ and $\psi(\xi)$. Due to symmetry of the problem, the potential function $\phi(\xi)$ and $\psi(\xi)$ take the form

$$
\begin{aligned}
& \phi(\xi)=\sum_{k=1}^{\infty} \frac{a_{k}}{\xi^{2 k-1}} \\
& \psi(\xi)=\sum_{k=1}^{\infty} \frac{b_{k}}{\xi^{2 k-1}}-\bar{\omega}(\xi) \frac{\phi^{\prime}(\xi)}{\omega^{\prime}(\xi)}
\end{aligned}
$$

The unknown coefficients $a_{k}$ and $b_{k}$ can be obtained using the boundary collocation method. In order to check the accuracy of the boundary collocation method, we only apply a remote biaxial loading. The calculated results are identical with the exact solution by Muskhelishvili[16] with eight digits accuracy.

Fig. 8 shows the normal stress along the extension line from the blunted crack tip for the case of $\tau_{f} / \mu=0.002, r_{o b} / b=5000, \alpha=45^{\circ}, \beta=60^{\circ}$, and $K_{I}^{t i p} / \mu \sqrt{2 \pi b}=0.218$. As the applied loading $\sigma_{0}$ increases, more dislocation are emitted. These emitted dislocations produce the back stress and image stress. It is clear that the effective $K$ stress field agrees very well with the present calculation results given by boundary collocation method at the distance larger than $300 \mathrm{~b}$. The effective $K$ field used here is defined as

$$
\sigma_{i j}=\frac{K_{I}^{+i p}}{\sqrt{2 \pi r}}+\sigma_{i j}^{d}
$$

On the other hand, the effective $K$ field is remarkable higher than that the accurate calculation results near the top of the notcl. As the applied load is high enough, the peak $\sigma_{\max }$ of the normal stress $\sigma_{22}$ will shift to some distance away from the notch tip. In this situation, the FDZ size is about $10 \mathrm{~b}$. It means that the strong interaction between emitted dislocations and the blunted crack will change the stress distribution pattern.

This feature becomes more pronounced when the materials resistance $K_{I e}$ for dislocation emission is small and the obstacle disappears. The hoop stress versus distance from the crack tip is plotted in Fig.9 for the case of $\tau_{f} / \mu=0.002, r_{o b} / b=\infty, \alpha=45^{\circ}, \beta=60^{\circ}$, and $K_{I}^{\text {tip }} / \mu \sqrt{2 \pi b}=0.1$.

\section{Conclusion Remarks}

Based on Peierls framework, the dislocation emission along a pair of symmetric slip planes from a Mode I crack tip were analyzed in this paper. The equilibrium positions and the total number of emitted dislocations were determined. The competition process between dislocation emission and quasi-cleavage is revealed. The interaction between the cohesive zone and the emitted dislocation results in gradually increase of the resistance $K_{I e}$, the critical stress intensity factor for a new dislocation emission. As the $K_{I}^{\text {tip }}>K_{I}^{\text {intrin }}$ the crack cleavage will occur. When the 
total number of emitted dislocations is large enough, the DFZ size becomes small enough, the hoop stress peak will shift to some distance away from the blunted crack tip. This may lead to the nucleation of a nanocrack in DFZ.

\section{Acknowledgements}

This work was supported by The National Nature Science Foundation of the China.

\section{References}

1. Ohr, S.M., Mater. Sci. Eng., 72(1985),1.

2. Horton J.A. and Ohr, S.M., Scripta Metal.,16(1982),621.

3. Chiao, Y.H. and Clark, D.R., Acta. Metall, 37(1989),203.

4. Rice, J.R. and Thomson, R.M., Phil. Mag., 29(1974),73.

5. Rice, J.R., J. Mech. Phys. Solids 40(1992),239.

6. Schoeck, G., Phil. Mag. 63(1991),111.

7. Rice, J.R., Beltz and Sun,Y., In topics in Fracture and Fatigue, A.S. Argon(ed.) Springer-Verlag (1992),1.

8. Wang, T.C., Int. J. Fracture, 69(1995),295.

9. Wang, T.C., Int. J. Fracture, 78(1996),227.

10. Wang, T.C., Philosophical Magazine A, 74(1996),983.

11. Chen, O. Chu, W. and Hsiao, C., Scripta metall. mater., 30(1994),1335.

12. Zhang, Y. Wang, Y. Chu, W. and Hsiao, C., Scripta metall. meter., 31(1994), 279.

13. Zhu, T. Yang, W. and Guo, T., Acta mater., 44(1996),3049.

14. Lin, I.H. and Thomson, R., Acta metall., 34(1986),187.

15. Suo, Z., Int. J. Solids Struct., 25(1989),1133.

16. Muskhelishvili, N. I., Some basic problem in the Mathematical Theory of Elasticity, Noordhoff, Groningen,1953.

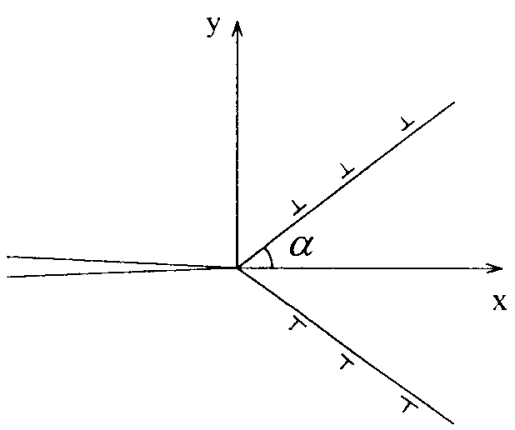

Fig.1 A pair of symmetric slip planes emanate from the crack tip. 

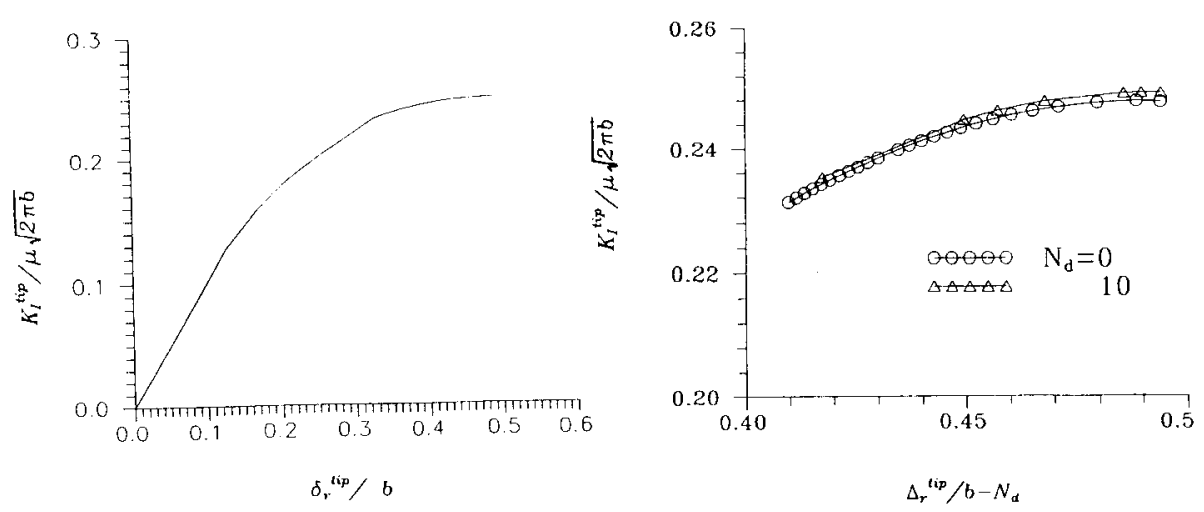

Fig. 2 Local stress intensity factor variation:(a). $K_{I}^{\text {tip }}$ versus $\delta_{\mathrm{r}}$ before dislocation emission;(b). $K_{t}^{\text {tip }}$ versus $\Delta_{\mathrm{r}}^{\text {tip }} / \mathrm{b}-\mathrm{N}_{\mathrm{d}}$ after the $\mathrm{N}_{\mathrm{d}}$-th dislocation emission.

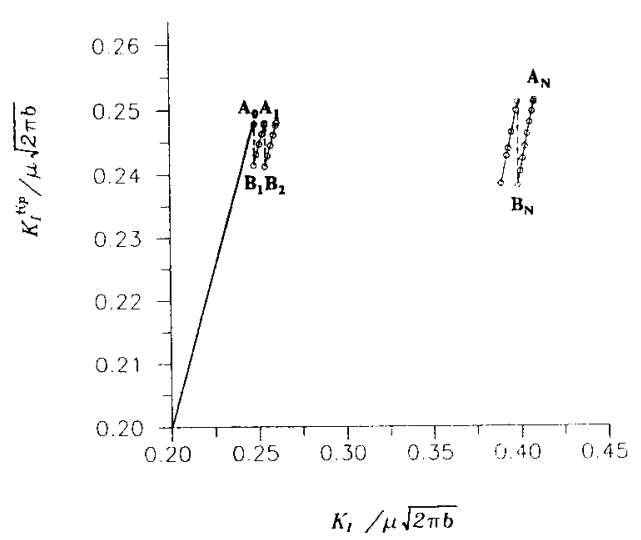

Fig. 3 Local stress intensity factor $K_{I}^{\text {tip }}$ versus applied stress intensity factor $K_{I}$.

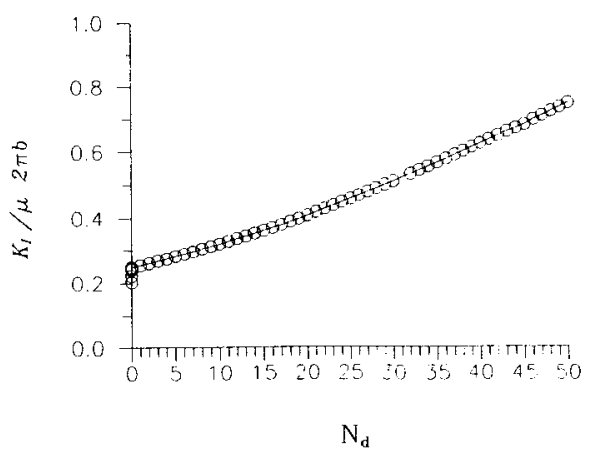

Fig. 5 Upper envelope of applied stress intensity factor $K_{I}$ versus emitted dislocation $\mathrm{N}_{\mathrm{d}}$.

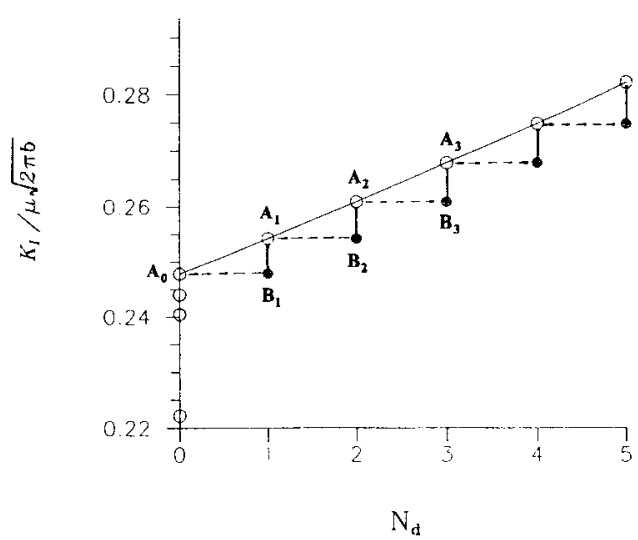

Fig.4 Applied stress intensity factor $K_{I}$ as a function of emitted dislocation $\mathrm{N}_{\mathrm{d}}$.

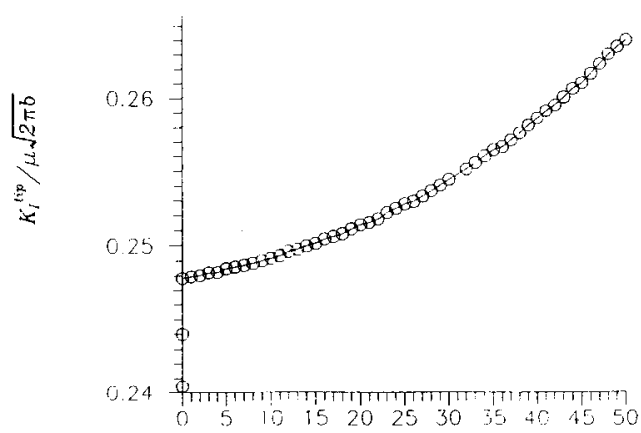

$\mathrm{N}_{\mathrm{a}}$

Fig.6 Upper envelope of local stress intensity factor $K_{l}^{t i p}$ versus emitted dislocation $\mathrm{N}_{\mathrm{d}}$. 


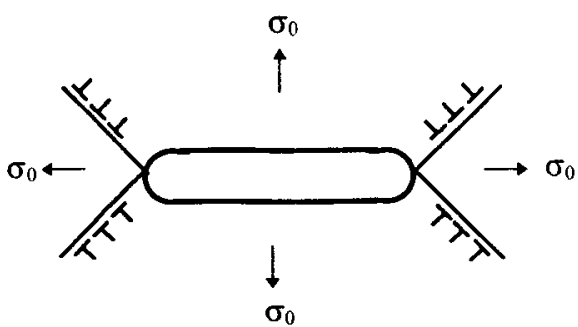

(a)

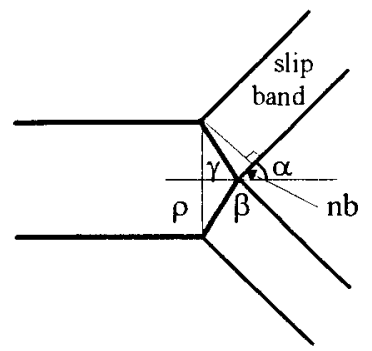

(b)

Fig.7 (a).Infinite plate with blunted crack, (b). A simplified slip model.

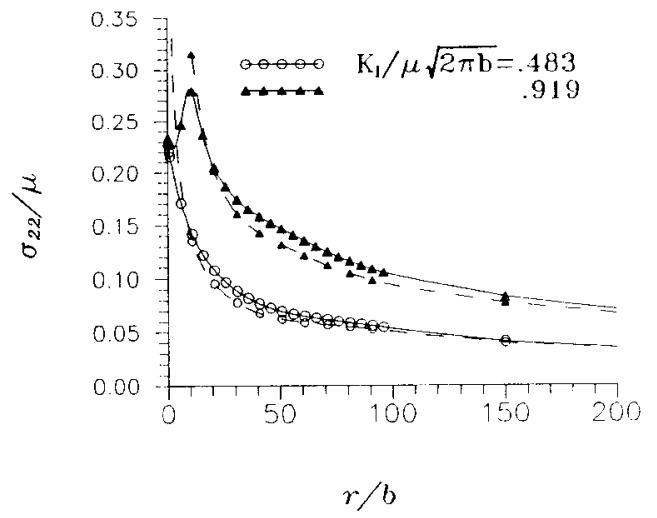

Fig. 8 Hoop stress distribution along the extension line of the blunted crack tip. The solid line are the accurate results, and the dashed line are the effective $\mathrm{K}$ field.

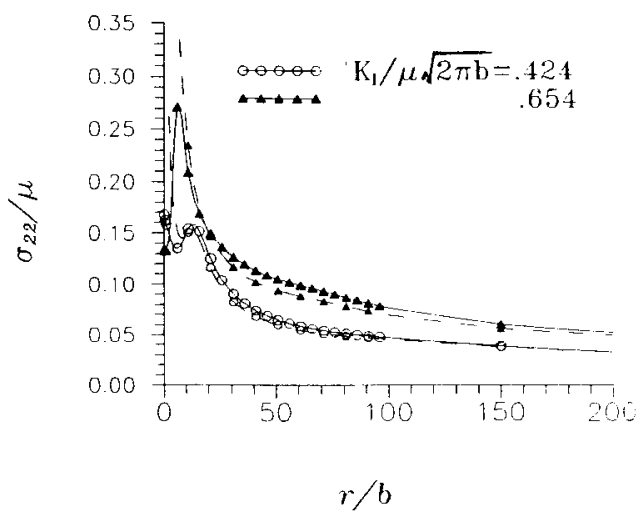

Fig.9 Hoop stress distribution for materials with lower resistance $\mathrm{K}_{\mathrm{Ie}}$ and without obstacle. 


\section{Fracture and Strength of Solids III}

10.4028/www.scientific.net/KEM.145-149

Dislocation Emission and Cleavage Process at Crack Tip

10.4028/www.scientific.net/KEM.145-149.113 